\title{
In Situ Detection of Particle Aggregation on Electrode Surfaces
}

\author{
Kamonwad Ngamchuea, Kristina Tschulik, Shaltiel Eloul, Richard G. Compton*
}

*corresponding author: Richard G. Compton, Department of Chemistry, Physical \& Theoretical Chemistry Laboratory, University of Oxford, South Parks Road, Oxford, OX1 3QZ, United Kingdom

Email: richard.compton@chem.ox.ac.uk. Tel: +44 (0) 1865275957 Fax: +44 (0) 1865 275410

\section{Key Words:}

Partially blocked electrodes; non-conductive nanoparticles; electrochemical sizing; chronoamperometry; numerical simulation

\begin{abstract}
Partially blocked electrodes (PBEs) are important; many applications use non-conductive nanoparticles (NPs) to introduce new electrode functionalities. As aggregation is a problem in NP immobilization, developing an in situ method to detect aggregation is vital to characterise such modified electrodes. We present chronoamperometry as a method for detection of NP surface aggregation and semi-quantitative sizing of the formed aggregates, based on the diffusion limited current measured at PBEs as compared with the values calculated numerically for different blocking feature sizes. In contrast to voltammetry, no approximations on electrode kinetics are needed, making chronoamperometry a more general and reliable method. Sizing is shown for two modification methods. Upon drop casting, significant aggregation is observed, while it is minimized in electrophoretic NP deposition. The aggregate sizes determined are in semi-quantitative agreement with ex situ microscopic analysis of the PBEs.
\end{abstract}




\section{$1 \quad$ Introduction}

Immobilization of particles on macroscopic electrodes is an important way to introduce selected surface functionalities, such as to target specific analytes or selectively catalyse reactions ${ }^{[1]}$. Notably, the beneficial use of particle modified electrodes is not limited to electroactive particles, but numerous reports exist on the modification of electrodes with electrochemically inert particles, of both nano- ${ }^{[2]}$ and micrometers ${ }^{[3]}$ in size.

In contrast to what one might expect intuitively, this partial blocking of the electrode is not directly reflected in a decrease in the measured current, but is largely dependent on the diffusion regime. At electrodes modified with nano- to micrometric particles, the current response depends on the size of the particles and the distance between adjacent particles relative to the diffusion layer thickness, $\sqrt{D t}$, where $t$ is the timescale of the experiment and $D$ is the analyte diffusion coefficient. For small particles, at sufficiently long time when the spacing between adjacent features becomes small compared to $\sqrt{D t}$, there is strong overlap of diffusional fields resulting in Cottrell currents where the electrode response reflects its geometric area ${ }^{[4]}$. With increasing size of the blocking features, this overlap of diffusional fields becomes less efficient and ultimately results diffusion currents which reflect only the extent of the unblocked surface ${ }^{[5]}$.

Aggregation of (nano)particles upon surface immobilization, especially during the most convenient technique of drop casting, is not only disadvantageous for most electrochemical applications, but identifying/quantifying the extent of aggregation is crucial to understand and correctly interpret the electrochemical response of such electrodes. An in situ approach to sizing such aggregates would be advantageous as it allows for a fast, reliable and inexpensive screening of different nanoparticle modification techniques for any electrode/nanoparticle system of interest.

Up until now, the effects of the partial blocking of electrodes on their electrochemical response have been studied using cyclic voltammetry ${ }^{[4 b, 5-6]}$. It has been found that the associated apparent decrease in the electrochemical rate constant of a redox process, for instance the oxidation of hexacyanoferrate (II) to hexacyanoferrate (III), can be used to determine the surface coverage of the electrode ${ }^{[4 f]}$ and under certain constraints, even the size of the blocking features ${ }^{[5]}$. Notably, these cyclic voltammetry approaches require the electrode kinetics to be known and sufficiently fast in order to access the size of the blocking 
features. It has been pointed out recently, ${ }^{[7]}$ that such assumptions may cause significant errors and shall therefore best be avoided.

Therefore, we herein present chronoamperometry as a simple electrochemical method to semi-quantitatively determine the size of blocking features on an electrode in situ. No approximations regarding the electrochemical kinetics are needed, as by applying a sufficiently high overpotential mass transport controlled experimental conditions are ensured and thus the size of the blocking features may be probed directly. Thus, we demonstrate the in situ detection of surface aggregation of non-conductive nanoparticles and the semiquantitative sizing of the resulting blocking features by comparing the current measured during chronoamperometric oxidation of hexacyanoferrate (II) anions in aqueous solution at bare and partially blocked glassy carbon electrodes with the values calculated numerically for various sizes of blocking features. Partially blocked electrodes are prepared by immobilizing $\mathrm{Fe}_{3} \mathrm{O}_{4}$ nanoparticles on the electrodes using two common modification methods, drop casting and electrophoresis. In accordance with literature ${ }^{[8]}$, distinctly different blocking feature sizes are found during chronoamperometric in situ sizing, showing large aggregates for the former and almost non-aggregated particles for the latter modification technique. Both chronoamperometrically determined blocking feature sizes are shown to be semi-quantitative by ex situ conventional electron microscopy, validating the applicability of the presented novel in situ approach.

\section{Theory}

Throughout we consider a 1-electron oxidation of the form,

$$
\mathrm{A} \rightleftarrows \mathrm{B}+\mathrm{e}^{-}
$$

in which only species ' $A$ ' is initially present in the solution. In a chronoamperometric experiment, the potential at the electrode is changed instantaneously from some initial potential, $E_{\mathrm{i}}$, which usually corresponds to zero current, to the potential, $E$, that usually corresponds to diffusion controlled oxidation of ' $\mathrm{A}$ ' and it is held at this value for a set time $t$. It is assumed that the system is under diffusion control and contributions from migration and convection to mass transport can be neglected. This is reasonable for experiments run for relatively short times $(10 \mathrm{~s})$, in the absence of mechanical stirring and the presence of excess 
supporting electrolyte, as done in this work. Both bare and partially blocked disc electrodes (PBEs) can be modelled using a full cylindrical geometry numerical simulation ${ }^{[9]}$ and simplified using the 'diffusion domain' approximation proposed by Gileadi ${ }^{[10]}$ and Amatore $^{[4 f]}$. According to that a PBE can be thought of as an array of inert particles of the same size regularly distributed over the electroactive electrode surface ${ }^{[11]}$. We assume that each particle is in an identical environment and so this array of particles can be divided into identical unit cells arranged in a hexagonal array ${ }^{[12]}$ (see Fig. 1 (a)). Using the diffusion domain approximation, these hexagonal unit cells can be approximated as cylindrical cells of the same base areas, as shown in Fig. 1 (b). It is only necessary to simulate a single diffusion domain. The results for the whole surface are then calculated by multiplying the results of a single unit cell by the number of such cells.

The area of a hexagon, $A_{\mathrm{h}}$, is given by:

$$
A_{\mathrm{h}}=\frac{\sqrt{3}}{2} d^{2}
$$

where $d$ is the height of the hexagon (or the centre-to-centre distance). The radius of the cylindrical cell, $r_{\mathrm{d}}$, is therefore determined from:

$$
\pi r_{\mathrm{d}}^{2}=\frac{\sqrt{3}}{2} d^{2}
$$

SO

$$
r_{\mathrm{d}} \approx 0.525 d
$$

a)

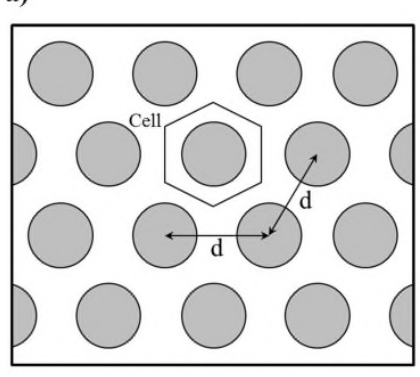

b)

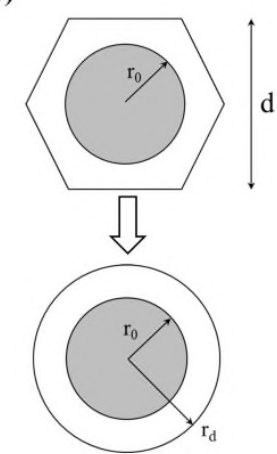

Figure 1: a) An array of hexagonal unit cells and

b) their transformation into cylindrical cells, where $\mathrm{r}_{0}$ is the radius of the blocking feature and $r_{d}$ is the radius of a diffusion domain.

Davies et al. ${ }^{[5]}$ found that particles on a surface might be reasonably approximated as flat discs, as compared to the diffusion layer thickness, the 'height' of a particle is small. The 
cylindrical unit cell may therefore be modelled using cylindrical polar coordinates as shown in Figure 2.

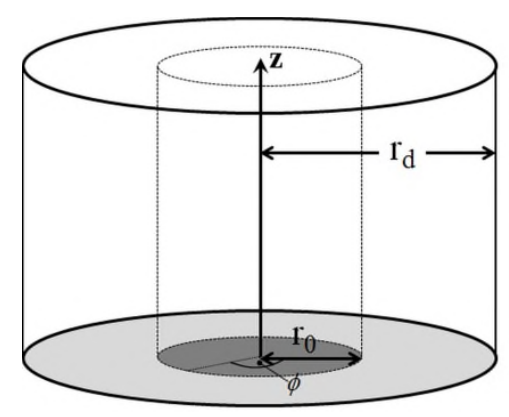

Figure 2: Cylindrical polar coordinate system for the diffusion domain unit cell sketched in Fig. 1b.

\subsection{Boundary Conditions}

\subsubsection{2-D Simulations of Chronoamperograms}

Only species ' $A$ ' is initially present and the solution is assumed to be uniform at the start of a simulation. Therefore, the starting bulk concentrations of species $i, c_{i}^{*}$, are as follows:

$$
\begin{aligned}
& c_{\mathrm{A}}(t=0)=c_{\mathrm{A}}^{*} ; \\
& c_{\mathrm{B}}(t=0)=c_{\mathrm{B}}^{*}=0 ;
\end{aligned}
$$

As has been shown previously ${ }^{[13]}$ that limiting the edge flux to a finite value in the simulations of chronoamperometry at zero surface coverage will not perturb the results of chronoamperometric experiments at suitably high overpotentials.

At sufficiently high overpotentials, resulting in complete consumption of A immediately after it reaches the electrode surface ${ }^{[14]}$.

The concentration of $\mathrm{A}$ at the electrode surface is therefore set to zero at all times:

$$
c_{\mathrm{A}}(z=0)=0
$$

The time-dependent mass transport of a chemical species $i$ due to diffusion across the unblocked surface is described by Fick's second law: 


$$
\frac{\partial c_{i}}{\partial t}=D_{i} \nabla^{2} c_{i}
$$

where $c_{i}$ and $D_{i}$ are the concentration and diffusion coefficient of species ' $i$ ' respectively.

At the surface of the blocking features, $0<r<r_{0}$, the flux of material through the surface is zero, i.e.

$$
\left.\frac{\partial c_{i}}{\partial z}\right|_{z=0}=0
$$

At the vertical axis $(r=0)$ and the edge of the diffusion domain $\left(r=r_{\mathrm{d}}\right)$, there are symmetry boundaries and so there can be no flux through these axes:

$$
\begin{aligned}
& \left.\frac{\partial c_{i}}{\partial r}\right|_{r=0}=0 ; \\
& \left.\frac{\partial c_{i}}{\partial r}\right|_{r=r_{\mathrm{d}}}=0 ;
\end{aligned}
$$

In the $z$ direction normal to the electrode surface (shown in Fig. 2), the size of the space must exceed the distance reachable by diffusion from the electrode over the timescale of the experiment to avoid computational errors. In one dimension, the root mean squared displacement of a particle from its starting position, $\sqrt{\overline{z^{2}}}$ (or $\bar{z}$ ), is equal to

$$
\bar{z}=\sqrt{2 D t}
$$

Therefore, to ensure no significant changes in concentration can propagate as far as the boundary, we must set the maximum simulation distance to be at some distance greater than $\bar{z}$. A typical value is ${ }^{[12]}$

$$
z_{\max }=6 \sqrt{D t_{\max }}
$$

where $t_{\max }$ is the maximum time the experiment will run for. Since there will be no change in concentration at $z=z_{\max }$, we can set the concentration of each species at this distance to the bulk value:

$$
c_{i}\left(z=z_{\max }\right)=c_{i}^{*}
$$

The boundary conditions for the 2-D simulations of chronoamperograms are summarized in Table 2 and shown schematically in Fig. 3. 


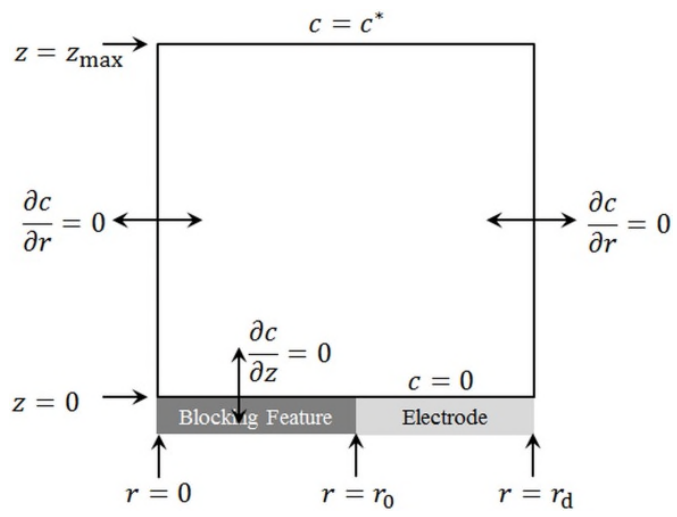

Figure 3: Schematic drawing of the two-dimensional simulation space and the boundary conditions used

\subsubsection{1-D Simulations of Cyclic Voltammograms}

The simulations of cyclic voltammograms are done in one-dimension, it is therefore not necessary to divide the electrode surface into unit cells. The boundary conditions required are the starting bulk concentrations of species A and B which are set according to Eqn. 2.5 and 2.6 and the electrode kinetics. At the surface of the electrode, the following Butler-Volmer boundary condition is used to set the concentration gradient across the surface ${ }^{[15]}$;

$$
\begin{aligned}
& k_{\mathrm{f}}=k^{0} \exp \left\{\frac{(1-\alpha) \mathrm{F}\left(E(t)-E_{\mathrm{f}}\right)}{\mathrm{R} T}\right\} ; \\
& k_{\mathrm{b}}=k^{0} \exp \left\{\frac{-\alpha \mathrm{F}\left(E(t)-E_{\mathrm{f}}\right)}{\mathrm{R} T}\right\} ;
\end{aligned}
$$

in which $k_{\mathrm{f}}$ is the electron transfer rate constant for the oxidation of $\mathrm{A}, k_{\mathrm{b}}$ is the electron transfer rate constant for the reduction of $\mathrm{B}, k^{0}$ is the standard electrochemical rate constant, $\alpha$ is the transfer coefficient as defined by IUPAC ${ }^{[16]}, \mathrm{F}$ is the Faraday constant, $E(t)$ is the potential at time $t, E_{\mathrm{f}}$ is the formal potential, $\mathrm{R}$ is the molar gas constant and $T$ is the temperature. Thus eqn. 2.7 is replaced for $r_{0}<r<r_{\mathrm{d}}$ by

$$
\left.D_{\mathrm{A}} \frac{\partial c_{\mathrm{A}}}{\partial z}\right|_{z=0}=k_{\mathrm{f}} c_{\mathrm{A}}(z=0)-k_{\mathrm{b}} c_{\mathrm{B}}(z=0)
$$

and

$$
\left.D_{\mathrm{A}} \frac{\partial c_{\mathrm{A}}}{\partial z}\right|_{z=0}=-\left.D_{\mathrm{B}} \frac{\partial c_{\mathrm{B}}}{\partial z}\right|_{z=0}
$$

where $D_{i}$ is the diffusion coefficient of species $i$ and $c_{i}$ is the concentration of species $i$. 


\section{Experimental Section}

\subsection{Chemical Reagents}

Potassium hexacyanoferrate (III), $\quad \mathrm{K}_{3}\left[\mathrm{Fe}(\mathrm{CN})_{6}\right], \quad 98+\% \quad$ (Lancaster), potassium hexacyanoferrate (II) trihydrate, $\mathrm{K}_{4}\left[\mathrm{Fe}(\mathrm{CN})_{6}\right] \cdot 3 \mathrm{H}_{2} \mathrm{O}, 99 \%$ (Lancaster) and potassium nitrate (Sigma-Aldrich) were used as received, without further purification. Hydrochloric acid (Sigma-Aldrich, >37\%) was diluted to the concentration of $0.1 \mathrm{M}$. All solutions were prepared using deionised water (Millipore) with a resistivity of $18.2 \mathrm{M} \Omega \mathrm{cm}$ at $25{ }^{\circ} \mathrm{C}$.

$\mathrm{Fe}_{3} \mathrm{O}_{4}$ NPs were made based on the method reported by Lyon et al. ${ }^{[17]}$ by dissolving $10 \mathrm{mmol}$ iron (II) chloride tetrahydrate $\left(\mathrm{FeCl}_{2} \cdot 4 \mathrm{H}_{2} \mathrm{O}\right.$, Sigma-Aldrich) and $20 \mathrm{mmol}$ iron (III) chloride hexahydrate $\left(\mathrm{FeCl}_{3} \cdot 6 \mathrm{H}_{2} \mathrm{O}\right.$, Sigma-Aldrich) in $27 \mathrm{~mL}$ solution of $0.8 \mathrm{M}$ hydrochloric acid ( $\mathrm{HCl}$, Sigma-Aldrich). The co-precipitation of $\mathrm{Fe}_{3} \mathrm{O}_{4}$ nanoparticles (NPs) was achieved at room temperature by adding $250 \mathrm{~mL}$ of a $1.7 \mathrm{M}$ aqueous solution of sodium hydroxide $(\mathrm{NaOH})$ and stirring the reaction mixture for 30 minutes. Afterwards, $\mathrm{Fe}_{3} \mathrm{O}_{4} \mathrm{NPs}$ were separated from the reaction mixture with the help of an $\mathrm{NdFeB}$ permanent magnet. $\mathrm{Fe}_{3} \mathrm{O}_{4}$ NPs were then washed with deionised water for three times. Then $3.3 \mathrm{~g}$ of these NPs were suspended in $135 \mathrm{~mL} \mathrm{H}_{2} \mathrm{O}$ and $3.5 \mathrm{~g}$ sodium citrate tribasic dihydrate was added to introduce citrate as a capping agent before the formation of the shell. This suspension was heated to $100{ }^{\circ} \mathrm{C}$ for 30 minutes with constant stirring and was left to cool to room temperature. The derived citrate-capped $\mathrm{Fe}_{3} \mathrm{O}_{4} \mathrm{NPs}$ were washed with deionised water and separated from the suspension using a permanent magnet. The nanoparticles were then suspended in $\mathrm{H}_{2} \mathrm{O}$ so as to yield a NP concentration of $3.5 \mathrm{~g} \mathrm{~L}^{-1}$. The size of the used citrate-capped $\mathrm{Fe}_{3} \mathrm{O}_{4} \mathrm{NPs}$ was determined using transmission electron microscope and was analysed in the Supporting Information to yield the radius of $4.0 \pm 1.0 \mathrm{~nm}$. The result is also in very good agreement with that reported by Lyon et al. ${ }^{[17]}$

\subsection{Instrumental}

Electrochemical experiments were performed in a thermostated $\left(25.0 \pm 0.2{ }^{\circ} \mathrm{C}\right)$ Faraday cage, using a $\mu$ Autolab Type III potentiostat (Utrecht, Netherlands). All measurements were conducted using a standard three-electrode setup utilising a carbon rod counter electrode (3 mm diameter) and a saturated calomel reference electrode (SCE, BASi, West Lafayette, IN, USA). A bare or modified glassy carbon electrode (GCE, $3 \mathrm{~mm}$ diameter) was employed as the working electrode. Prior to use, the working electrode was polished using 1.0, 0.3 and 
$0.05 \mu \mathrm{m}$ alumina powder (Buehler) on soft lapping pads (Buehler), then sonicated in deionised water in an ultrasonic bath for 1 minute to remove any adsorbed material.

Simulations were performed with a Dell Precision T3610 with two quad-core hyperthreaded Intel® Xeon ${ }^{\circledR}$ E5-1620 processors $(3.70 \mathrm{GHz})$.

\subsection{Experimental Procedures}

\subsubsection{Bare Electrodes}

Cyclic voltammetry (CV) scans were run in aqueous solutions of $9.5 \mathrm{mM}\left[\mathrm{Fe}(\mathrm{CN})_{6}\right]^{4-}$ with $0.50 \mathrm{M} \mathrm{KNO}_{3}$ supporting electrolyte, sweeping potential from $-0.2 \mathrm{~V}$ to $+0.7 \mathrm{~V}$ and back to 0.2 V. All potentials were measured against an SCE and scan rates of 25, 50, 100, 200, 300 and $400 \mathrm{mVs}^{-1}$ were employed.

Chronoamperometric experiments were run for 10 seconds in the same electrolyte solution as for CVs. A potential of $0.35 \mathrm{~V}$ vs SCE was applied to ensure mass transport controlled conditions.

\subsubsection{Partially Blocked Electrodes}

\section{- Modifications of Electrode Surfaces with NPs}

Electrodes were modified via two approaches: drop casting and electrophoretic particle deposition $^{[8 \mathrm{a}],[8 \mathrm{~b}]}$. Both methods have been reported ${ }^{[8]}$ to yield different sizes of surface features for similar surface coverages caused by different extents of aggregation on electrode surface; typically, drop-casting yields much bigger aggregate sizes. This allows us to vary the size of $\mathrm{Fe}_{3} \mathrm{O}_{4}$ particle blocking sites at a constant total coverage. The surface coverage was chosen such that it is high enough to give rise to changes in electrochemical responses, but not too high that there would be the possibility of blocking the entire electrode surface. A surface coverage of ca. 50-60\% was therefore chosen.

\section{Drop Casting}

Glassy carbon electrodes were modified by dropping $2 \mu \mathrm{L}$ of the $1 / 200$ diluted citratedcapped $\mathrm{Fe}_{3} \mathrm{O}_{4}$ NPs suspension onto the surface and letting the electrode dry under nitrogen gas flow. The volume and concentration of NPs suspension used correspond to $\sim 60 \%$ coverage considering the NP size and its initial concentration. Three independent repeats of 
electrode modification by this method were performed and the experimental results provided in the text represent the mean and the standard deviation of the obtained data.

\section{Electrophoretic Particle Immobilization}

A positive potential was applied to the glassy carbon working electrode immersed in the NP suspension so that the citrate-capped $\mathrm{Fe}_{3} \mathrm{O}_{4}$ nanoparticles (with a negative zeta potential of ca. $-34 \mathrm{mV}$ ) were electrophoretically deposited on the electrode surface. The potential applied was chosen such that the electrodes and NPs were inert in the NPs suspension. A platinum mesh and a silver wire were used as counter- and reference- electrodes respectively. In order to get a surface coverage similar to that obtained via drop casting ( $60 \%)$, potential of $0.2 \mathrm{~V}$ vs Ag wire was applied for 10 minutes. Three independent repeats of electrode modification by this method were performed and the experimental results provided in the text represent the mean and the standard deviation of the obtained data.

\section{- Electrochemical Stripping Experiments}

Electrochemical stripping experiments of modified electrodes subjected to linear sweep voltammetry at a scan rate of $10 \mathrm{mV} \mathrm{s}^{-1}$ in $0.1 \mathrm{M} \mathrm{HCl}$ solution were used to quantify the amount of NPs immobilized on the electrode surface by measuring the charge associated with their stripping from the electrode ${ }^{[18]}$.

\section{- Cyclic Voltammetry and Chronoamperometry}

Cyclic voltammetry and chronoamperometry were performed in the same manner as for the bare electrode, but modified electrodes were instead utilised as working electrodes.

\subsection{Simulations}

\subsubsection{1-D Simulations of Cyclic Voltammograms}

The transient responses for an oxidation process: $\mathrm{A} \rightleftarrows \mathrm{B}+\mathrm{e}^{-}$, characterised by a formal potential, $E_{\mathrm{f}}$, transfer coefficient, $\alpha$ (as defined by IUPAC ${ }^{[16]}$ ) and a standard rate constant, $k^{0}$, were simulated using BASi DigiSim® software version $3.03 b^{[19]}$. Simulations of cyclic voltammograms were performed starting with bulk concentrations of $\mathrm{A}$ and $\mathrm{B}$ of $9.5 \mathrm{mM}$ and $0 \mathrm{mM}$ respectively. The diffusion coefficients of $A$ and $B$ are $7.53 \times 10^{-10} \mathrm{~m}^{2} \mathrm{~s}^{-1}$ and 
$8.40 \times 10^{-10} \mathrm{~m}^{2} \mathrm{~s}^{-1}$ at $25^{\circ} \mathrm{C}$ respectively; in agreement with the values reported for similar electrolytes $^{[20]}$.

\subsubsection{2-D Simulations of Chronoamperograms}

Simulations were performed using the software package COMSOL Multiphysics ${ }^{\circledR}$ version $5.0^{[21]}$. Convergence studies were run for both linear and two-dimensional systems according Kätelhön et al. ${ }^{[22]}$ to yield the current transients of less than $0.5 \%$ difference from the Randles-Sevcik equation and the Shoup-Szabo equation respectively, to determine optimum parameters for spatial and temporal grid densities which gave a good compromise between runtime and simulation accuracy. The simulation space is divided into small finite spatial grids called a mesh. This mesh is arranged such that there is a greater density of grid points where rapid and large changes in concentration are expected, most notably at the surface of a particle and the electrode surface. A 'boundary layer' is hence used to create a greater density of grid points in these regions, while a 'free quad' mesh is used throughout the remaining simulation space with an expansion ratio of 1.2 in the z-direction starting from $z=0$.

The simulation model is normalized using the set of dimensionless parameters shown in the SI. This normalization reduces the number of simulations required and the resulting chronoamperograms can conveniently be scaled to apply to any set of $r_{\mathrm{d}}$ values ${ }^{[12]}$.

\subsection{SEM Analysis}

SEM imaging was performed to size the blocking features generated by drop casting and electrophoretic deposition of $\mathrm{Fe}_{3} \mathrm{O}_{4}$ nanoparticles on glassy carbon electrodes. Therefore, glassy carbon plates were modified as described above and imaged using a Leo Gemini II Field emission gun microscope (Zeiss, Germany).

\section{$4 \quad$ Results and Discussion}

\subsection{Experimental Results}

In order to develop an in situ approach to detect surface agglomeration of non-conductive nanoparticles on an electrode surface, two modification methods, drop casting and electrophoretic particle deposition, were used to modify glassy carbon electrodes. These two 
methods are known to give rise to largely different feature sizes for the same surface coverage used due to different extent of surface aggregation. As reported in literatures ${ }^{[8 \mathrm{~d}, 23]}$, drop casting typically results in strong surface aggregation upon the evaporation of the solvent and therefore a significantly bigger size of blocking features is formed, as compared to NP immobilization by electrophoretic deposition which particle aggregation is minimized $^{[8 b, 8 c]}$. The surface coverage of the thus prepared partially blocked electrodes is then first determined by two independent methods, namely electrochemical stripping experiments (section 4.1.1.) and cyclic voltammetry (sections 4.1.2 and 4.2.1). Second, the formation of nanoparticle aggregates is evidenced and the size of features blocking the electrodes is estimated semi-quantitatively in situ using chronoamperometry (sections 4.1.3 and 4.2.2). Third, the in situ inferred sizes are verified by ex situ SEM imaging to prove the applicability of the developed electrochemical method.

\subsubsection{Surface Coverage}

In order to compare the electrochemical responses of the electrodes modified by different methods, all other parameters including the electrode surface coverages should be as similar as possible. Surface coverages were determined using two independent techniques: electrochemical stripping experiments and the cyclic voltammetry approach developed by Amatore et al. ${ }^{[4 f]}$ The surface coverage agreed by the two techniques will then be used as a fixed parameter for the following experiments and simulations of chronoamperograms, in which we seek to develop a general in situ technique to determine the size of blocking features on electrodes.

\section{Electrochemical stripping experiments}

Linear sweep voltammetry of modified glassy carbon electrodes from $0.60 \mathrm{~V}$ to $-0.50 \mathrm{~V}$ vs $\mathrm{SCE}$ at a scan rate of $10 \mathrm{mVs}^{-1}$ in $0.1 \mathrm{M} \mathrm{HCl}$ solution was run and repeated until no further reductive peak was observed. The integrals of current over time give the values of total charges required to reductively strip the $\mathrm{Fe}_{3} \mathrm{O}_{4}$ NPs (see Eqn. 4.2) from the glassy carbon electrode. These total charges $(Q)$ were found to be $96 \pm 8 \mu \mathrm{C}$ and $105 \pm 10 \mu \mathrm{C}$ for electrodes modified by drop casting and electrophoresis respectively. 

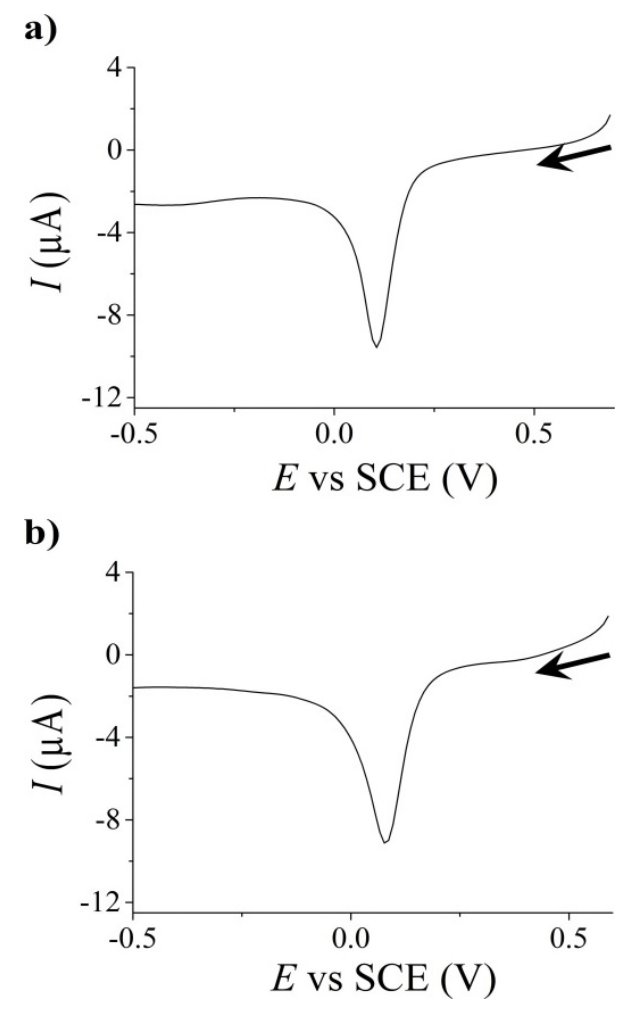

Figure 4: Linear sweep voltammograms in $0.1 \mathrm{M} \mathrm{HCl}$ utilising glassy carbon electrodes modified with $\mathrm{Fe}_{3} \mathrm{O}_{4}$ NPs via a) drop casting, b) electrophoretic particle deposition

Faraday's law of electrolysis relates the total charge, $Q$, to the number of species undergoing electrochemical processes, $n$ :

$$
Q=n z \mathrm{~F}
$$

where $z$ is the number of transferred electrons per formula unit.

According to the chemical equation below, the value of $z=2$ for the reduction of $\mathrm{Fe}_{3} \mathrm{O}_{4}$ can be taken. ${ }^{[24]}$

$$
\mathrm{Fe}_{3} \mathrm{O}_{4}(\mathrm{~s})+8 \mathrm{H}^{+}(\mathrm{aq})+2 \mathrm{e}^{-} \longrightarrow 3 \mathrm{Fe}^{2+}(\mathrm{aq})+4 \mathrm{H}_{2} \mathrm{O}(\mathrm{l})
$$

In order to calculate the surface coverage, the amount of $\mathrm{Fe}_{3} \mathrm{O}_{4}$ in moles $\left(N_{\mathrm{NP}}\right)$ in one spherical nanoparticle was calculated using Equation 4.3:

$$
N_{\mathrm{NP}}=\frac{\rho V_{\mathrm{NP}}}{M_{r}}
$$


where $V_{\mathrm{NP}}$ is the volume of a nanoparticle $\left(V_{\mathrm{NP}}=\frac{4}{3} \pi r_{\mathrm{NP}}^{3}\right), \rho$ is the density of $\mathrm{Fe}_{3} \mathrm{O}_{4}$ $\left(\rho=5.1 \times 10^{3} \mathrm{kgm}^{-3}\right)^{[25]}$ and $M_{r}$ is the molar mass of $\mathrm{Fe}_{3} \mathrm{O}_{4}\left(M_{r}=0.2315 \mathrm{kgmol}^{-1}\right)^{[25]}$. According to TEM analysis, the mean radius of a $\mathrm{Fe}_{3} \mathrm{O}_{4}$ nanoparticle is $4.0 \mathrm{~nm}$ (see $\mathrm{SI}$ ), therefore $N_{\mathrm{NP}}=5.9 \times 10^{-21}$ moles.

The number of NPs immobilized on the electrode surface, $N$, can be calculated using Equation 4.1 coupled with the value of $N_{\mathrm{NP}}$ :

$$
N=\frac{n}{N_{\mathrm{NP}}}=\frac{n}{5.9 \times 10^{-21}}
$$

Thus, the surface coverage, $\theta$, follows as:

$$
\theta=\frac{N \pi r_{\mathrm{NP}}^{2}}{\pi r_{\mathrm{e}}^{2}}
$$

where $N$ is the number of nanoparticles in moles, $r_{\mathrm{NP}}$ is the nanoparticle radius and $r_{\mathrm{e}}$ is the electrode radius. The surface coverage can thus be calculated to $60 \pm 10 \%$ for both drop casting and electrophoretic modifications. Therefore, $\theta=60 \%$ is used as a fixed parameter in all the following experiments. Likewise, $\theta=60 \%$ is used to calculate the relative size of $r_{0}$ and $r_{\mathrm{d}}$ in numerical simulations in section 4.2.2.

\subsubsection{Cyclic Voltammetry}

To study the effects of the modification of glassy carbon electrodes with citrate-capped $\mathrm{Fe}_{3} \mathrm{O}_{4}$ particles on voltammetric responses, cyclic voltammograms (CVs) at varied scan rates in 9.5 $\mathrm{mM}\left[\mathrm{Fe}(\mathrm{CN})_{6}\right]^{4-}$ with fully supporting $\mathrm{KNO}_{3}$ electrolyte were obtained for bare electrodes and electrodes modified by drop casting, as shown in Fig. 5. These CVs at varied scan rates are also used to determine the electrode surface coverage; this will be explained explicitly in Section 4.2.1.

Compared with the bare electrode, the CVs of the PBE show an increased peak-to-peak separation and slightly smaller peak currents at all scan rates (see Fig. 5). These changes in the cyclic voltammograms mean that a potential larger than thermodynamically required was needed to bring about the oxidation of $\left[\mathrm{Fe}(\mathrm{CN})_{6}\right]^{4-}$ anions in the forward scan and the reduction of $\left[\mathrm{Fe}(\mathrm{CN})_{6}\right]^{3-}$ in the reverse $\mathrm{scan}^{[14]}$. In other words, the process appears more electrochemically irreversible. This apparent slowing down of kinetics suggests that the 
citrated-capped $\mathrm{Fe}_{3} \mathrm{O}_{4}$ nanoparticles are much less electroactive than the glassy carbon electrode, and so act as particles blocking the electrode surface ${ }^{[4 f]}$.
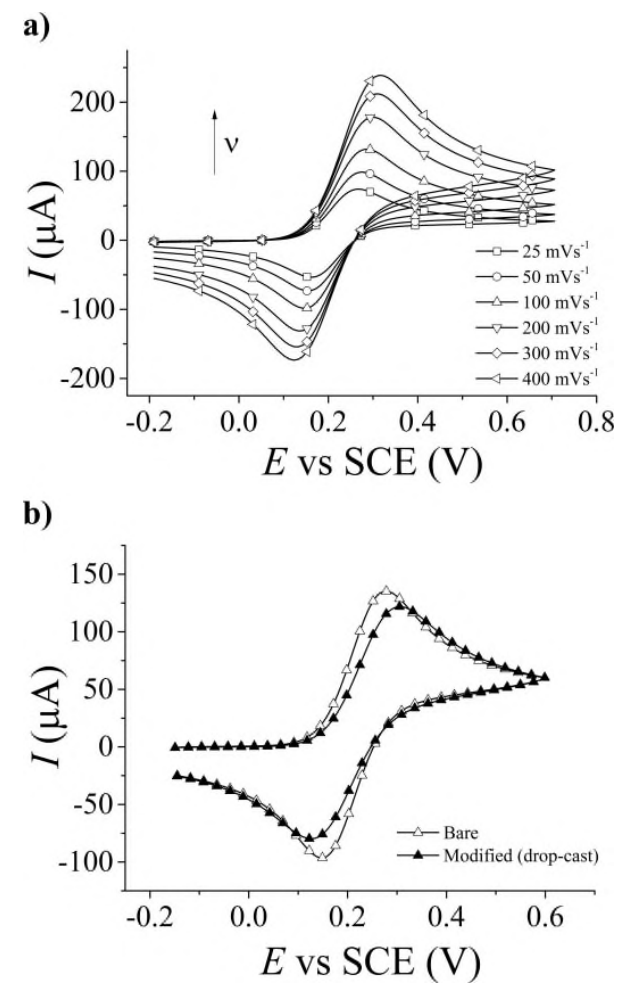

Figure 5: Cyclic voltammograms in $9.5 \mathrm{mM}\left[\mathrm{Fe}(\mathrm{CN})_{6}\right]^{4-}+0.5 \mathrm{M} \mathrm{KNO}_{3}$ aqueous solutions utilising a) bare GC at varied scan rates, b) bare GC and modified (drop cast) GC at the scan rate of $100 \mathrm{mVs}^{-1}$

\subsubsection{Chronoamperometry}

Chronoamperometry was performed experimentally to observe the current transients for bare and modified electrodes. Chronoamperometry was run at a sufficiently high over-potential $\left(0.35 \mathrm{~V}\right.$ vs SCE) such that all $\left[\mathrm{Fe}(\mathrm{CN})_{6}\right]^{4-}$ ions which arrive at the electrode were oxidized. The concentration of this species at the electrode surface is therefore effectively zero at all but ultra-short times and the system was under diffusion control. Consequently, chronoamperometry can be used to deliberately separate the influence of electrode kinetics.

Chronoamperograms obtained for the bare and the two types of modified electrodes under mass-transport limited conditions are shown in Fig. 6. Each experiment was repeated at least three times. The average currents and their standard deviations measured at $t=10 \mathrm{~s}$ for bare, drop-cast modified and electrophoretically modified electrodes were $34.1 \pm 0.1 \mu \mathrm{A}$, $33.6 \pm 0.1 \mu \mathrm{A}$ and $34.1 \pm 0.2 \mu \mathrm{A}$ respectively. Thus, compared with the bare electrode, experimental results show no significant change in currents when the electrode was modified 
via electrophoresis. In contrast, a $\sim 1.5 \%$ decrease in currents was observed when electrodes were modified via drop casting.

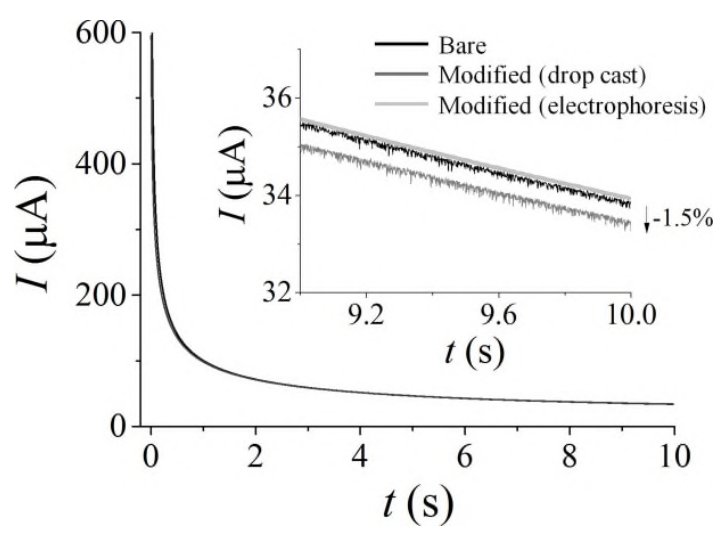

Figure 6: Chronoamperometric measurements in $9.5 \mathrm{mM}\left[\mathrm{Fe}(\mathrm{CN})_{6}\right]^{4-}+0.5 \mathrm{M} \mathrm{KNO}_{3}$ aqueous solutions at $E=0.35 \mathrm{~V}$ vs SCE

The reduction in currents observed in the drop casting experiments supports the conclusion that the citrated-capped $\mathrm{Fe}_{3} \mathrm{O}_{4}$ nanoparticles are essentially non-conductive and voltammetrically inert and hence blocking parts of the electrode surface. However this decrease in currents was not observed for electrodes modified by electrophoretic deposition where the same particles and the same surface coverage were used.

If the particles were nanometre in size as expected from the size of nanoparticles used, no change in electrochemical response would be observed since the very effective overlap of adjacent diffusional fields leads, in effect, to apparent one-dimensional diffusion to the electrode as a whole with a current response reflecting the electrode geometry not the unblocked surface. As the particle size increases, the overlap of diffusional fields is reduced $^{[11 c]}$. Therefore, it can be concluded that the bigger difference in currents relative to the bare electrode of the drop casting case is because of significant aggregation of nanoparticles on the electrode surface, resulting in a bigger particles size.

These characteristics of chronoamperometric signals for different block features of equal electrode surface coverage will be studied in detail in section 4.2.2 to gain information not only of the amount of blocking particles, but also the size of these blocking sites from numerical simulation of chronoamperometry. 


\subsection{Simulations}

\subsubsection{1-D Simulations of Cyclic Voltammograms}

The cyclic voltammograms obtained from experiments were fitted by numerical simulations of the hexacyanoferrate(II)/hexacyanoferrate (III) redox couple using the parameters described in Section 3.4.1. The diffusion coefficients were obtained experimentally using a platinum micro electrode and were in good agreement with literature values as mentioned in Section 3.4.1. The transfer coefficient which best fit with both the bare and the modified electrodes is $\alpha=0.5$. Using this value of $\alpha$, the best fit rate constants for all experimental scan rates are $k^{0}=0.0029 \mathrm{~cm} \mathrm{~s}^{-1}$ for the bare electrode, and for the electrode modified by drop casting, a smaller apparent rate constant of $k_{\mathrm{app}}^{0}=0.0012 \mathrm{~cm} \mathrm{~s}^{-1}$ results.

a)
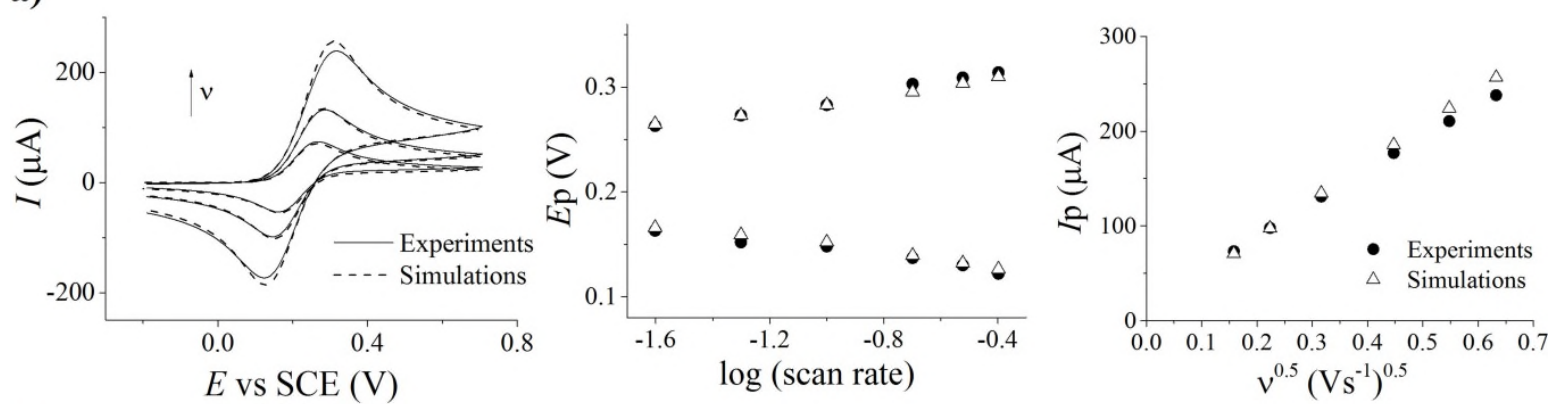

b)
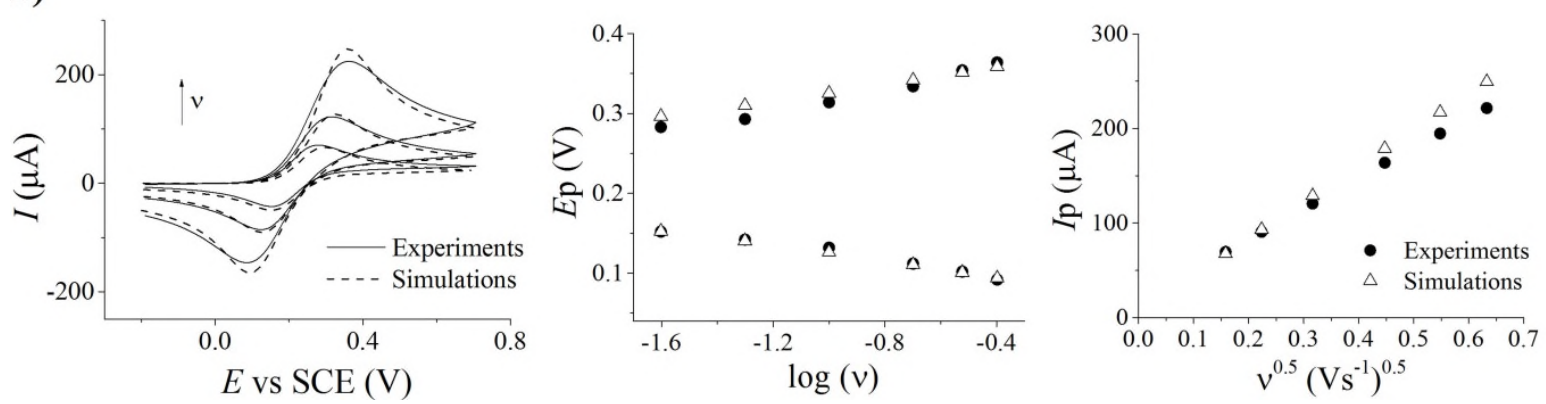

Figure 7: (Left): cyclic voltammograms $\left(v=25,100\right.$ and $\left.400 \mathrm{mVs}^{-1}\right)$ in $9.5 \mathrm{mM}\left[\mathrm{Fe}(\mathrm{CN})_{6}\right]^{4-}$ + $0.5 \mathrm{M} \mathrm{KNO}_{3}$ aqueous solutions utilising a) bare $\mathrm{GC}$, b) drop cast $\mathrm{Fe}_{3} \mathrm{O}_{4} / \mathrm{GC}$; (Middle and Right): the plots of peak potentials and peak currents obtained from experiments vs simulations

According to Amatore et al. ${ }^{[4 f]}$, surface coverages can be determined from this apparent rate constant, $k_{\mathrm{app}}^{0}$, of a PBE using Eqn. 4.6, assuming that the blocking features are flat and that 
they are closely spaced resulting in strongly overlapping diffusional fields reflecting 1-D linear diffusion

$$
k_{\mathrm{app}}^{0}=k^{0}(1-\theta)
$$

Using this approach, the surface coverage, $\theta$, for drop casting was found to be $58 \%$, which is in excellent agreement with electrochemical stripping experiments (where $\theta \sim 60 \%$ was determined).

Note that without further assumptions, the size of blocking particles cannot be determined from simulations of cyclic voltammetry ${ }^{[5]}$. A novel approach, a combination of experiments and 2-dimensional simulations of chronoamperograms, is therefore developed in the following section to enable semi-quantitative sizing of the blocking sites.

\subsubsection{2-D Simulations of Chronoamperograms}

To study the effect of the particle size on time-dependent electrochemical response at a potentiostated electrode, the variation of the size of inert particles, $r_{0}$, was investigated. Equivalently, this was done by varying the size of diffusion domains, $r_{\mathrm{d}}$ (for simulations of a single unit cell and surface coverage of $\theta=60 \%$ ).

The simulated sizes of $r_{\mathrm{d}}$ are $5.5 \mathrm{~nm}, 1 \mu \mathrm{m}, 2 \mu \mathrm{m}, 100 \mu \mathrm{m}, 200 \mu \mathrm{m}, 500 \mu \mathrm{m}, 1 \mathrm{~mm}$ and $1.47 \mathrm{~mm}$. These values are chosen such that the transition of diffusional regimes from 2dimensional type (for small $r_{\mathrm{d}}$ ) to 1-dimensional type (for large $r_{\mathrm{d}}$ ) can be investigated. Once we gain the knowledge of how chronoamperometric currents vary with the size of blocking features, we can then use this information to determine the size of these blocking features in situ.

The dimensional transient current for a single unit cell is determined by Eqn. 4.7;

$$
I=\left.2 \pi \mathrm{F} D_{\mathrm{A}} c_{\mathrm{A}}^{*} r_{\mathrm{d}} \int_{R=0}^{R=1} \frac{\partial c_{i}}{\partial Z}\right|_{Z=0} R \mathrm{~d} R
$$

where the integral term is the dimensionless current for a 2-dimensional simulation space obtained from numerical simulations using COMSOL and the factor $2 \pi$ comes from integrating about the angle $\phi$ (shown in Fig. 2). 
The current for the whole surface, $I_{\text {total }}$, is then calculated using:

$$
I_{\text {total }}=I \cdot \frac{r_{\mathrm{e}}^{2}}{r_{\mathrm{d}}^{2}}
$$

where $I$ is dimensional current for a single unit cell, $r_{\mathrm{e}}$ is the electrode radius $(1.47 \mathrm{~mm}), r_{\mathrm{d}}$ is the radius of the diffusion domain and $\frac{r_{\mathrm{e}}^{2}}{r_{\mathrm{d}}^{2}}$, therefore, quantifies the number of unit cells.

The resulting current-time curves are plotted in Fig. 8 (a) and in Fig. 8 (b), the currents at a time of $t=10 \mathrm{~s}$ are compared for different size of blocking features.

a)
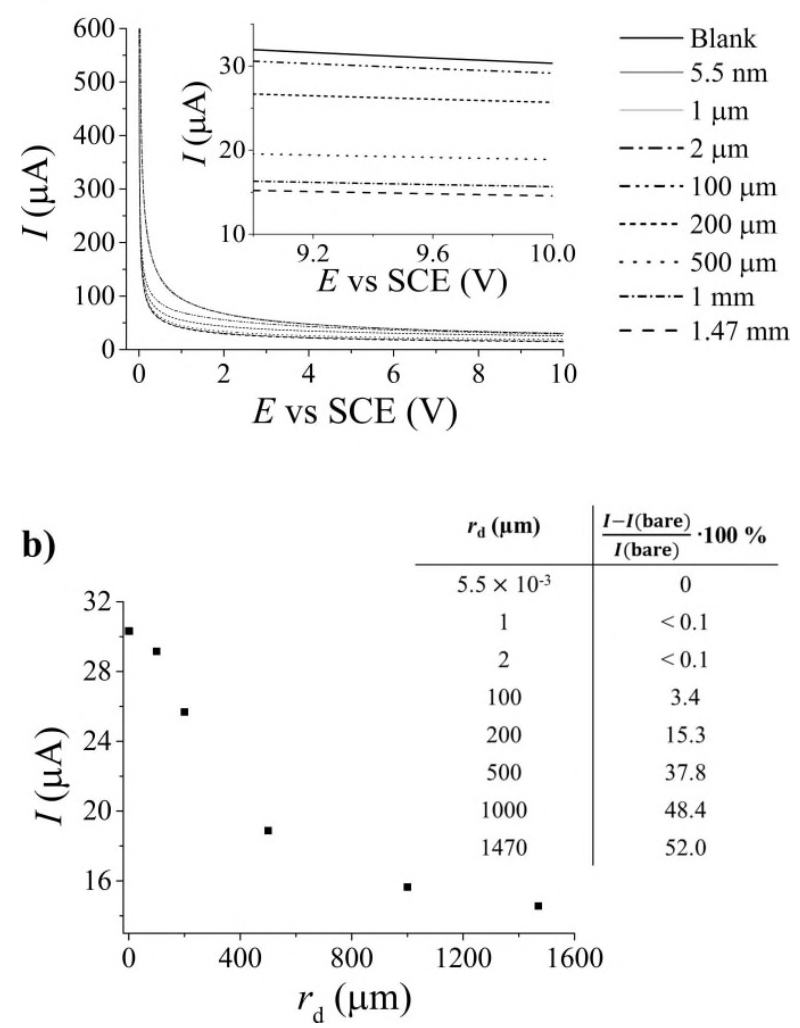

Figure 8: a) chronoamperometric simulations for electrodes blocked by features of different sizes,

b) currents at $t=10 \mathrm{~s}$ for electrodes blocked by features of different sizes; $\theta=60 \%$

It can be seen that the current decreases for increasing feature sizes due to the less effective overlap of adjacent diffusional fields, resulting in a current which reflects the smaller effective electrode surface area i.e. from the whole electrode geometry (active+inert) for small features to only the unblocked surface for large features. Comparison of the experimental results with this simulated data shows that the measured decrease of the current of $1.5 \%$ at $t=10 \mathrm{~s}$ for the drop-cast modification corresponds to a blocking feature size of 
about $40 \mu \mathrm{m}$ in radius. The negligible effect of electrophoretic nanoparticle deposition on the chronoamperometric current confirms that particle sizes resulting from this modification method are smaller than $2 \mu \mathrm{m}$. The information we obtained from simulations combined with the experimental results hence suggests and quantifies increased particle aggregation during drop casting as compared to electrophoretic electrode modification, which is in excellent agreement with literature reports using ex situ microscopic characterization of electroactive NPs ${ }^{[8 b, 8 d]}$.

\subsection{Confirmation of Size and Distribution}

To verify the results of the chronoamperometric detection of nanoparticle surface aggregation and the semi-quantitative sizing of blocking features, an ex situ SEM analysis of glassy carbon plates modified by drop casting and electrophoresis are performed. The surface modified by drop casting shows a high level of inhomogeneity as shown in Fig. 9 (a). The observed feature sizes range from about 40 to $100 \mu \mathrm{m}$ in radius. This is in very good semiquantitative agreement with the value estimated from numerical simulation of the chronoamperometric response. This confirms the ability to not only evidence the formation of surface aggregates upon modification of electrodes with nanoparticles, but also shows that semi-quantitative sizing of the formed blocking features can be achieved by chronoamperometric means.

The surface modified by electrophoresis shows improved homogeneity and a smaller particle size of several nanometers in radius, which agrees with the experimental results. As obtained from the simulation, for particles smaller than $2 \mu \mathrm{m}$, the change in chronoamperometric currents is less than $1 \%$, making it not possible to detect within the experimental errors and thus only allows the semi-quantitative estimation of a maximum feature size. 
a)

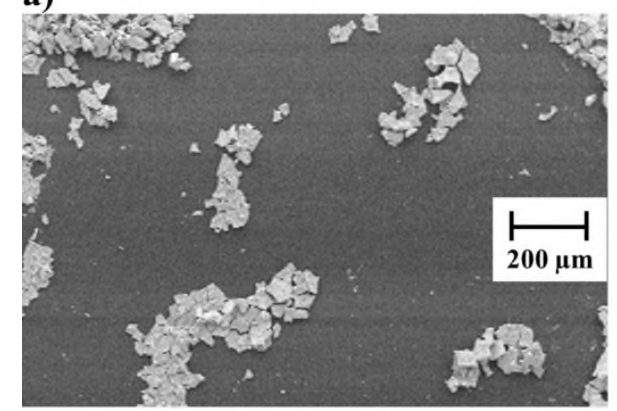

b)

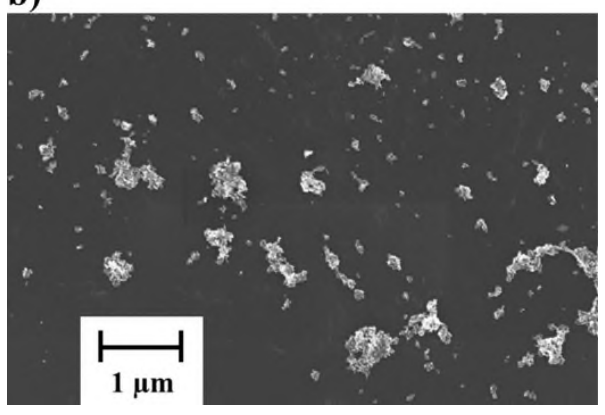

Figure 9: SEM images of glassy carbon electrodes modified with $\mathrm{Fe}_{3} \mathrm{O}_{4} \mathrm{NPs}$ by a) drop casting, b) electrophoresis

\section{Conclusions}

A method has been developed that allows the semi-quantitative in situ sizing of nonconducting features on an electrode surface using chronoamperometry, a simple, reliable and inexpensive electrochemical technique. This approach enables estimating the amount of aggregation occurring during nanoparticle immobilization on electrode surfaces, which is crucially important for the electrochemical response of such electrodes. Importantly - and in contrast to existing voltammetric methods for characterising partially blocked surfaces - no assumptions or approximations regarding the electrode kinetics of a reaction and the diffusional overlap between neighbouring blocking sites has to be made. This makes the developed approach original and much more generally applicable.

The herein in situ observed large difference in the resulting feature size upon surface immobilization of nanoparticles on electrode surfaces during two of the most common modification methods, emphasizes the demand for such semi-quantitative in situ characterization techniques, as due to the inherent sensitivity of nanoparticle immobilization approaches, the quality of the produced modified electrodes have to be monitored, which is time consuming and expensive when done via conventional ex situ microscopic techniques. 


\section{Acknowledgements}

We thank Dr. Veronika Hähnel (IFW Dresden, Germany) for SEM imaging and Hendriette Van der Walt (MINTEK, Randburg, South Africa) for assistance during $\mathrm{Fe}_{3} \mathrm{O}_{4}$ nanoparticle preparation. Christine Damm (IFW Dresden, Germany) is acknowledged for TEM characterisation of the obtained nanoparticles. KN acknowledges funding from the Royal Thai government under the Development and Promotion of Science and Technology Talents Project. KT was supported by a Marie Curie Intra European Fellowship under the FP 7 Framework Programme [no. 327706]. RGC acknowledges funding from the ERC Grant Agreement [no. 320403].

\section{References}

[1] X. Luo, A. Morrin, A. J. Killard, M. R. Smyth, Electroanalysis 2006, 18, 319-326.

[2] aJ. Bao, C. Hou, Y. Zhang, Q. Li, D. Huo, M. Yang, X. Luo, J Electrochem Soc 2014, 162, B47-B51; bD. Cao, P. He, N. Hu, The Analyst 2003, 128, 1268-1274; cY. Zhang, P. He, N. Hu, Electrochimica Acta 2004, 49, 1981-1988.

[3] aW. Hou, Talanta 1991, 38, 557-560; bY. H. Li, W. S. Huang, Anal Methods-Uk 2015, 7, 2537-2541.

[4] aT. J. Davies, C. E. Banks, R. G. Compton, Journal of Solid State Electrochemistry 2005, 9, 797-808; bB. A. Brookes, T. J. Davies, A. C. Fisher, R. G. Evans, S. J. Wilkins, K. Yunus, J. D. Wadhawan, R. G. Compton, The Journal of Physical Chemistry B 2003, 107, 1616-1627; cT. J. Davies, B. A. Brookes, A. C. Fisher, K. Yunus, S. J. Wilkins, P. R. Greene, J. D. Wadhawan, R. G. Compton, The Journal of Physical Chemistry B 2003, 107, 6431-6444; dF. G. Chevallier, T. J. Davies, O. V. Klymenko, L. Jiang, T. G. J. Jones, R. G. Compton, J Electroanal Chem 2005, 577, 211-221; eT. J. Davies, R. R. Moore, C. E. Banks, R. G. Compton, J Electroanal Chem 2004, 574, 123-152; fC. Amatore, J. M. Savéant, D. Tessier, Journal of Electroanalytical Chemistry and Interfacial Electrochemistry 1983, 147, 39-51.

[5] T. J. Davies, E. R. Lowe, S. J. Wilkins, R. G. Compton, Chemphyschem : a European journal of chemical physics and physical chemistry 2005, 6, 1340-1347.

[6] aC. A. Amatore, M. R. Deakin, M. Wightman, Journal of Electroanalytical Chemistry and Interfacial Electrochemistry 1986, 206, 23-36; bM. R. Deakin, R. M. Wightman, 
C. A. Amatore, Journal of Electroanalytical Chemistry and Interfacial

Electrochemistry 1986, 215, 49-61; cC. A. Amatore, B. Fosset, M. R. Deakin, R. M. Wightman, Journal of Electroanalytical Chemistry and Interfacial Electrochemistry 1987, 225, 33-48; dT. J. Davies, B. A. Brookes, R. G. Compton, J Electroanal Chem 2004, 566, 193-216; eO. Sliusarenko, A. Oleinick, I. Svir, C. Amatore, Electroanalysis 2015, 27, 980-991.

[7] A. N. Simonov, G. P. Morris, E. A. Mashkina, B. Bethwaite, K. Gillow, R. E. Baker, D. J. Gavaghan, A. M. Bond, Anal Chem 2014, 86, 8408-8417.

[8] aK. Tschulik, C. Batchelor-McAuley, H. S. Toh, E. J. Stuart, R. G. Compton, Physical chemistry chemical physics : PCCP 2014, 16, 616-623; bY. Wang, E. Laborda, B. J. Plowman, K. Tschulik, K. R. Ward, R. G. Palgrave, C. Damm, R. G. Compton, Physical chemistry chemical physics : PCCP 2014, 16, 3200-3208; cC. C. M. Neumann, E. Laborda, K. Tschulik, K. R. Ward, R. G. Compton, Nano Research 2013, 6, 511-524; dH. S. Toh, C. Batchelor-McAuley, K. Tschulik, M. Uhlemann, A. Crossley, R. G. Compton, Nanoscale 2013, 5, 4884-4893.

[9] E. J. F. Dickinson, J. G. Limon-Petersen, N. V. Rees, R. G. Compton, The Journal of Physical Chemistry C 2009, 113, 11157-11171.

[10] H. Reller, F. Kirowa-Eisner, E. Gileadi, Journal of Electroanalytical Chemistry and Interfacial Electrochemistry 1982, 138, 65-77.

[11] aS. R. Belding, R. G. Compton, Journal of Physical Chemistry C 2010, 114, 83098319; bF. W. Campbell, S. R. Belding, R. Baron, L. Xiao, R. G. Compton, The Journal of Physical Chemistry C 2009, 113, 9053-9062; cT. J. Davies, R. G. Compton, J Electroanal Chem 2005, 585, 63-82.

[12] R. G. Compton, E. Laborda, K. R. Ward, Understanding Voltammetry: Simulation of Electrode Processes, Imperial College Press, 2013.

[13] S. R. Belding, E. I. Rogers, R. G. Compton, The Journal of Physical Chemistry C 2009, 113, 4202-4207.

[14] R. G. Compton, C. E. Banks, Understanding Voltammetry, 2nd ed., Imperial College Press, 2011.

[15] aJ. A. V. Butler, Transactions of the Faraday Society 1924, 19, 729; bJ. A. V. Butler, Transactions of the Faraday Society 1924, 19, 734; cT. V. Erdey-Gruz, M., Z. physik. Chem. 1930, 150, 203-213.

[16] aR. Guidelli, R. G. Compton, J. M. Feliu, E. Gileadi, J. Lipkowski, W. Schmickler, S. Trasatti, Pure and Applied Chemistry 2014, 86, 245-258; bR. Guidelli, R. G. 
Compton, J. M. Feliu, E. Gileadi, J. Lipkowski, W. Schmickler, S. Trasatti, Pure and Applied Chemistry 2014, 86, 259-262.

[17] J. L. Lyon, D. A. Fleming, M. B. Stone, P. Schiffer, M. E. Williams, Nano letters 2004, 4, 719-723.

[18] H. S. Toh, C. Batchelor-McAuley, K. Tschulik, C. Damm, R. G. Compton, Sensors and Actuators B: Chemical 2014, 193, 315-319.

[19] A. W. Bott, S. W. Feldberg, M. Rudolph, Current Separation 1996, 15, 67-71.

[20] aS. J. Konopka, B. McDuffie, Anal Chem 1970, 42, 1741-1746; bK. Ngamchuea, S. Eloul, K. Tschulik, R. G. Compton, Journal of Solid State Electrochemistry 2014, 18, 3251-3257.

[21] E. J. F. Dickinson, H. Ekström, E. Fontes, Electrochemistry Communications 2014, 40, 71-74.

[22] aE. Kätelhön, R. G. Compton, The Analyst 2015, 140, 2592-2598; bE. Kätelhön, R. G. Compton, The Analyst 2015, 140, 3290.

[23] aR. Wallace, A. P. Brown, R. Brydson, S. J. Milne, N. Hondow, P. Wang, Journal of Physics: Conference Series 2012, 371, 012080; bJ. D. Torrey, T. L. Kirschling, L. F. Greenlee, Journal of Research of the National Institute of Standards and Technology 2015, 120,1 .

[24] Z. Y. Lu, D. M. Muir, Journal of Applied Electrochemistry 1986, 16, 745-756.

[25] W. M. Haynes, CRC Handbook of Chemistry and Physics (Internet Version 2015) 95th ed., CRC Press/Taylor and Francis, Boca Raton, FL. 


\section{Table of Contents}

\section{In Situ Detection of Particle Aggregation on Electrode Surfaces}

Kamonwad Ngamchuea, Dr. Kristina Tschulik, Shaltiel Eloul, Prof. Richard G. Compton

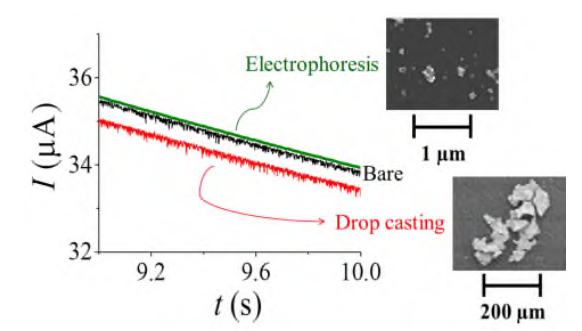

Using chronoamperometry, a new in situ method to semi-quantitatively size aggregates of non-conductive particles on electrode surfaces is developed. Importantly, the sensitivity of the electrochemical response of nanoparticle modified electrodes on the modification/immobilization technique used is highlighted. 